\title{
Impacts of produced water origin on bacterial community structures of activated sludge
}

\author{
Zhenyu Wang ${ }^{1,2}$, Feng Pan ${ }^{1}$, Abd El-Latif Hesham ${ }^{1,3}$, Yingxin Gao ${ }^{1, *}$, Yu Zhang ${ }^{1, *}$, \\ Min Yang ${ }^{1}$
}

1. State Key Laboratory of Environmental Aquatic Chemistry, Research Center for Eco-Environmental Sciences, Chinese Academy of Sciences, Beijing 100085, China. E-mail: zywang@htu.edu.cn

2. Key Laboratory for Microorganisms and Functional Molecules, College of Life Science, Henan Normal University, Xinxiang, Henan 453007, China

3. Genetics Department, Faculty of Agriculture, Assiut University, Assiut 71526, Egypt

\section{A R T I C L E I N F O}

Article history:

Received 6 November 2014

Revised 20 April 2015

Accepted 22 April 2015

Available online 21 August 2015

Keywords:

Polycyclic aromatic hydrocarbons

Indigenous community

Wastewater treatment

Functional gene

\begin{abstract}
A B S T R A C T
The purpose of this study was to reveal how activated sludge communities respond to influent quality and indigenous communities by treating two produced waters from different origins in a batch reactor in succession. The community shift and compositions were investigated using Polymerase Chain Reaction-denaturing gradient gel electrophoresis (PCR-DGGE) and further $16 \mathrm{~S}$ ribosomal DNA (rDNA) clone library analysis. The abundance of targeted genes for polycyclic aromatic hydrocarbon (PAH) degradation, nahAc/phnAc and C12O/C23O, was tracked to define the metabolic ability of the in situ microbial community by Most Probable Number (MPN) PCR. The biosystem performed almost the same for treatment of both produced waters in terms of removals of chemical oxygen demand (COD) and PAHs. Sludge communities were closely associated with the respective influent bacterial communities (similarity $>60 \%$ ), while one sludge clone library was dominated by the Betaproteobacteria (38\%) and Bacteriodetes (30\%) and the other was dominated by Gammaproteobacteria (52\%). This suggested that different influent and water quality have an effect on sludge community compositions. In addition, the existence of catabolic genes in sludge was consistent with the potential for degradation of PAHs in the treatment of both produced waters.
\end{abstract}

(c) 2015 The Research Center for Eco-Environmental Sciences, Chinese Academy of Sciences. Published by Elsevier B.V.

\section{Introduction}

During gas and oil extraction, a waste stream called produced water is generated, which contains relatively high concentrations of hydrocarbons including alkanes and polycyclic aromatic hydrocarbons (PAHs), and is in general treated with a biological process. Owing to their persistence and potential deleterious effects on the environment and human health,
PAHs have long been an important group of pollutants of environmental concern (Chowdhury et al., 2009).

Since the first PAH-degrading bacterial isolate was acquired from crude oil deposits 80 years ago, extensive studies have focused on the biodegradation of PAHs (Cerniglia, 1992). Bacterial groups belonging to Pseudomonas spp., Sphingomonas spp., Burkholderia spp., etc., have been found to be mainly responsible for the degradation of PAHs (Peng et al., 2008).

\footnotetext{
* Corresponding authors. E-mails: gyx@rcees.ac.cn (Yingxin Gao), zhangyu@rcees.ac.cn (Yu Zhang).
} 
With the development of culture independent molecular techniques, more candidate PAH degraders have been found in different environments contaminated with PAHs. At the same time, the genes encoding dioxygenase and catechol cleavage dioxygenase, such as the phnAc/nahAc and C12O/ C230 genes, have been confirmed to be the key functional genes during PAH mineralization. The functional gene abundance has been used as an indicator of PAH degradation ability at the community level (Tuomi et al., 2004).

Previous studies have shown that low molecular weight PAHs in produced waters were effectively removed through biological treatment (Wang et al., 2007). In such a system, high community similarity between the raw produced water and wastewater treating biomass was observed, suggesting the potential role of the indigenous bacterial community from the oil reservoir in the biodegradation of the hydrocarbons, including PAHs. It is known that the environmental conditions for different oil reservoirs, such as the temperature, oil compositions, salinity, etc., could be very diverse, which may lead to significant differences in indigenous bacterial communities. For example, in the high-temperature oil reservoirs in the North Sea oil-field, the microbial community was dominated by Firmicutes (about 55\%) with 20\% Bacteroidetes (Hakon et al., 2008), while Proteobacteria and Firmicutes were dominant in the produced water from mesophilic oil reservoirs (Pham et al., 2009), and a single phylotype of Arcobacter was found in an oil reservoir with low temperature (Grabowski et al., 2005). Since such a substantial difference in the indigenous bacterial communities existed in different oil reservoirs, it is interesting to know how the different indigenous bacterial communities will impact the sludge bacterial communities as well as the correlations among community composition, function and wastewater treatment performance of a biological system.

In this study, two different produced waters, one from HeBei oilfield (HBPW) and another from Xinjiang oilfield (XJPW), with different pollutant compositions, salinity and community composition, were used to evaluate the impacts of water quality and indigenous community on activated sludge using a batch reactor. The reactor was used to treat HBPW stably for two months before switching to treat XJPW. The community shift and compositions were revealed using PCR-DGGE (denature gradient gel electrophoresis) combined with 16S rDNA clone library analysis. At the same time, the sets of catabolic genes were monitored by Most Probable Number-Polymerase Chain Reaction (MPN-PCR) (Tuomi et al., 2004; Zhang et al., 2008) using primer sets for general detection of the genes encoding nahAc, phnAc, C23O, and C12O. The result of this study will allow a better understanding of the response of activated sludge communities to produced water quality and indigenous communities. The co-variation of the bacterial community and functional performance suggest a pathway to more efficient design and operation of produced water treatment processes.

\section{Materials and methods}

\subsection{Simulated system and sample collection}

A conventional aerobic sludge system was set up to evaluate the performance and microbial community composition and succession of activated sludge. The system was operated in a sequential batch reactor at $45 \pm 1^{\circ} \mathrm{C}$ and natural $\mathrm{pH}(7.2-7.4)$, the same as that of the existing full scale system. The reactor was mixed by aeration with HRT at 2-2.5 days and the organic loading rate ranged from $125-150 \mathrm{mg}$ chemical oxygen demand $(\mathrm{COD}) /(\mathrm{L} \cdot$ day). The reactor was started up with the initial biomass concentration of $4 \mathrm{~g} / \mathrm{L}$. Sludge volume was tracked as the indicator of biomass and sludge volume index (SVI) was $100 \mathrm{~mL} / \mathrm{g}$, along with stable effluent COD. Both the activated sludge and HBPW were taken from an existing oilfield bio-treatment system in eastern China. Both effluent and activated sludge were sampled every three days for COD, $\mathrm{PAH}$ and molecular biology analyses. After 8 weeks, with stable performance in terms of effluent COD, only the influent was substituted by another produced water XJPW with the same activated sludge and operating parameters as above. The effluent and activated sludge was also sampled every three days for PAH and molecular biology analysis for another 8 weeks until the effluent COD was stable. The last sludge samples for produced water $\mathrm{HB}$ as influent (HBPW clone library) and produced water XJ as influent (XJPW clone library) were chosen for clone analysis. Biomass was evaluated during the running process without discharge.

\subsection{Quantification of PAHs and water quality analysis}

Both influent and effluent water samples $(250 \mathrm{~mL})$ from HBPW and XJPW were taken for PAH analysis according to the protocol of the US Environmental Protection Agency (US EPA) and were processed immediately (Chen et al., 2005). The concentrations of PAHs in the extracts were determined by an Agilent 6890GC equipped with a 5973 mass selective detector using an HP-5 fused silica capillary column $(60 \mathrm{~m} \times 0.25 \mathrm{~mm} \times 0.25 \mu \mathrm{m}$ film thickness) under the selected ion monitoring mode. The recoveries for surrogate standards fell within a fairly narrow range and, for individual PAHs, were between $73.2 \% \pm 5.6 \%$ and $90.3 \% \pm 6.2 \%$.

The COD, total organic carbon (TOC), redox potential (ORP), and chlorate were determined, respectively, according to the standard methods (Andrew et al., 1998).

\subsection{Detection and enumeration of catabolic genes}

DNA templates from quantitative sludge samples $(0.1 \mathrm{~g})$ and influent water samples $(10 \mathrm{~mL})$ were independently extracted in triplicate and serially diluted 10-fold, and three samples of each dilution step were subjected to PCR. The MPN number was determined as described previously (Zhang et al., 2008), based on the cut-off probability theory of Kohno and Fukunaga (1998). The programs of touchdown PCR and primers of phnAc/nahAc, C12O/C230 and eubacteria 16S rRNA gene (rrs) were described in Wang et al. (2007).

\subsection{Cloning of PCR-amplified 16S rRNA genes and sequencing}

After primary screening of PCR-DGGE (Zhang et al., 2008; Wang et al., 2007), an activated sludge sample taken just before switching influent and the last one for XJPW treatment were subjected to clone analysis. Undigested PCR products amplified by primers $27 \mathrm{~F}$ (5'AGAGTTTGATCMTGGCTCAG3') and 1492R 
(5'TACGGY TACCTTGTTACGACT3') (Stevenson et al., 2011) containing 16S ribosomal RNA (rRNA) gene sequences from two activated sludge samples were visualized on a $1.2 \%$ agarose gel. Bands corresponding to the correct size $(1.5 \mathrm{~kb})$ were excised and purified using a DNA column purification kit (Sangon, China) according to the manufacturer instructions. The purified PCR products were then ligated into vector PGEM-T (Sangon, China). Ligation mixes were transformed into competent Escherichia coli JB-109. White colonies were picked randomly and cultured in $5 \mathrm{~mL}$ LB medium. About 90 clones from each sample with inserts of the right size were sent to the company for sequencing by primers M13F and M13R.

\subsection{Phylogenetic analysis}

Sequences obtained were initially submitted to the GenBank database (http://www.ncbi.nlm.nih.gov/) using the BLAST algorithm to roughly determine their phylogenetic affiliation. Partial sequences were aligned with the CLUSTALX program and phylogenetic dendrograms were constructed by the neighbor-joining method using the MEGA4 package.

\subsection{Nucleotide sequence Accession Numbers}

The sequence data reported in this study have been deposited in the Genbank database under Accession Nos. EF648015 to EF648107 for 16S rRNA gene clones from the sludge sample for HBPW treatment. The data for Accession Nos. EF648108 to EF648167 were obtained from the sludge sample for XJPW treatment.

\section{Results and discussions}

\subsection{Water quality and PAH distribution in two produced wastewaters}

The main water quality parameters of the two produced waters are shown in Table 1, and the distribution of PAHs in the two influents and effluents are compared in Table 2. HBPW exhibited a significantly high PAH concentration $(98.30 \mu \mathrm{g} / \mathrm{L})$ but a low

\begin{tabular}{|c|c|c|}
\hline Parameter & HBPW & XJPW \\
\hline TOC (mg/L) & $56 \pm 5$ & $49 \pm 5$ \\
\hline Total PAHs $(\mu \mathrm{g} / \mathrm{L})$ & 98.30 & 10.06 \\
\hline TDS (mg/L) & $5560 \pm 350$ & $16087 \pm 1200$ \\
\hline $\mathrm{Cl}^{-}(\mathrm{mg} / \mathrm{L})$ & $4159 \pm 320$ & $8491 \pm 520$ \\
\hline $\mathrm{pH}$ & 7.30 & 7.80 \\
\hline BTEX (mg/L) & $65 \pm 25$ & $62 \pm 20$ \\
\hline $\mathrm{SS}(\mathrm{mg} / \mathrm{L})$ & $30 \pm 5.5$ & $40 \pm 4$ \\
\hline $\mathrm{COD}(\mathrm{mg} / \mathrm{L}))$ & $270 \pm 20$ & $248 \pm 20$ \\
\hline Effluent COD (mg/L) & $75 \pm 10$ & $70 \pm 12$ \\
\hline
\end{tabular}

TDS: total dissolved solid; SS: suspended solid; BTEX: benzene, toluene, ethylbenzene, and xylenes. TOC: total organic carbon; PAHs: polycyclic aromatic hydrocarbons; COD: chemical oxygen demand.

Two different produced waters, one is from HeBei oilfield (HBPW) and another is from Xinjiang oilfield (XJPW).
Table 2 - Polycyclic aromatic hydrocarbon (PAH) distribution

and removal of two sources of produced water.

\begin{tabular}{lllll}
\hline \multicolumn{1}{c}{ Items } & $\begin{array}{c}\text { HBPW } \\
(\mathrm{ng} / \mathrm{L})\end{array}$ & Effluent & $\begin{array}{l}\text { XJPW } \\
(\mathrm{ng} / \mathrm{L})\end{array}$ & Effluent \\
\hline Nap and methyl-nap & 94665 & 310.4 & 7726.6 & 13.3 \\
FLU & 1182.6 & 52.4 & 582.2 & 28.3 \\
PHE & 1367.4 & 119.4 & 928.5 & 48.7 \\
ANA & 321.3 & 5.1 & 779.9 & 90.3 \\
FLT & 26.1 & 17.7 & 60.2 & 18.3 \\
ANT & 24.5 & 0 & 45 & 2.5 \\
PYR & 26.4 & 25.1 & 66.7 & 56.9 \\
ANY & 5.9 & 0 & 50.2 & 0 \\
BaA & 4.9 & 10.7 & 22.2 & 10.2 \\
CHR & 3.4 & 5.8 & 9.8 & 12.3 \\
BbFA & 6.2 & 2.2 & ND & ND \\
BkFA & 3.1 & 0 & ND & ND \\
BeP & 3.1 & 0 & ND & ND \\
PER & 10.6 & 0 & ND & ND \\
DBA & 1.5 & 0 & ND & ND \\
BPE & 3.7 & 4.6 & ND & ND \\
Total PAHs & 98300 & 553.4 & 10060 & 280.8 \\
Removal & $99.4 \%$ & & $97.2 \%$ & \\
\hline
\end{tabular}

ND: not detectable; FLU: fluorene; PHE:phenanthrene; ANA: anthracene; FLT: Fluoranthene; ANT: Anthracene; PYR: Pyrene; ANY: Acenaphthylene; BaA: Benzo(a)anthracene; CHR: Chrysene; BbFA: Benzo(b)fluoranthene; BkFA: Benzo(k)gluoranthene; BeP: Benzo(e)pyrene; PER: Perylene; DBA: Dibenzo(a.h)anthracene; BPE: Benzo(g,hi)perylene.

chloride $(4159 \pm 320 \mathrm{mg} / \mathrm{L})$ concentration, in comparison with XJPW $(10.06 \mu \mathrm{g} / \mathrm{L}$ and $8491 \pm 520 \mathrm{mg} / \mathrm{L}$, respectively). The other parameters including COD, TOC, and benzene, toluene, ethylbenzene, and xylenes (BTEX) were not significantly different. As for the distribution of PAHs, naphthalene and methylnaphthalene were dominant $(94665 \mathrm{ng} / \mathrm{L})$ followed by phenanthrene $(1367.4 \mathrm{ng} / \mathrm{L})$, fluorene $(1182.6 \mathrm{ng} / \mathrm{L})$ and anthracene (321.3 ng/L) in HBPW, while naphthalene and methylnaphthalene were dominant $(7726.6 \mathrm{ng} / \mathrm{L})$ followed by phenanthrene (928.5 ng/L), anthracene (779.9 ng/L) and fluorene (582.2 ng/L) in XJPW. Other common PAHs present like pyrene, benze[a] anthracene and chrysene had not much difference.

\subsection{Removal performance of simulated system}

As shown in Table 1, the reactor could remove the COD from $270 \pm 20 \mathrm{mg} / \mathrm{L}$ to $75 \pm 10 \mathrm{mg} / \mathrm{L}$ for HBPW, and from $248 \pm$ $20 \mathrm{mg} / \mathrm{L}$ to $70 \pm 12 \mathrm{mg} / \mathrm{L}$ for XJPW. This showed that the simulated system performed well for both produced waters in term of COD removal. The removals of PAHs for both produced waters were over $97 \%$, which was in accordance with the fullscale produced water treatment system (Wang et al., 2007). The concentrations of individual PAHs, including the 16 priority pollutants by US EPA, were detected for both influents and effluents. The different produced waters had obvious differences in $\mathrm{PAH}$ concentrations, both for lower and higher molecular weight (LMW/HMW) PAHs. Although the PAH removal rates for both produced waters were above $97 \%$, most PAH removal occurred in the LMW PAHs (Table 2), even though HMW PAHs have been found to be degraded by pure cultures and consortia (Dandie et al., 2004; Juhasz et al., 2000). It is possible that the bioavailability of HMW PAHs is lower than that 
of LMW PAHs and other hydrocarbons in real wastewater treatment. Thus the removal of HMW PAHs from actual wastewater is still a challenge for environmental protection.

\subsection{Shift of produced water treating microbial community}

The shift of the produced water treating community was tracked by PCR-DGGE targeting the V3 region of bacterial $16 \mathrm{~S}$ rDNA by comparison with the indigenous microbial communities of the two produced waters, respectively (data not shown). The similarity between HBPW and XJPW bacterial communities was very low (approximately 20\%). However, the HBPW sludge community exhibited a very high similarity with the indigenous bacterial community of influent HBPW (over 60\%), and a similar relationship was also observed between the XJPW sludge bacterial community and the indigenous bacterial community of influent XJPW (over 60\%). This result demonstrated that the indigenous bacterial communities of produced water and quality had exerted significant impacts on each sludge community, and may have played an important role in the degradation of the hydrocarbon pollutants.

To obtain more detailed information on the microbial composition, two 16S rRNA gene fragment libraries from sludge DNA samples were constructed by bacteria specific primer sets (HBPW clone library and XJPW clone library). Sequences with more than $97 \%$ similarity were regarded as one operational taxonomic unit (OTU) using DOTUR (Defining Operational Taxonomic Units and Estimating Species Richness) software. A total of 25 and 16 OTUs were achieved from 93 HB and 60 XJPW sludge bacterial clones, respectively. For the HBPW bacterial clone library (Fig. 1a), the Bacteriodetes and Betaproteobacteria amounted to 68\%, and the Alphaproteobacteria and Gammaproteobacteria amounted to $18 \%$ of the sequenced clones. These four groups dominated, amounting to more than $85 \%$, in HBPW clones. This clone library also contained OTUs that were members of the Spirochaetes (2\%), Firmicutes (4\%), Chlorobi $(4 \%)$, Planctomycetes (2\%) and Acidobacteria (2\%). For the XJPW bacterial clone library (Fig. 1b), the Bacteriodetes and Gammaproteobacteria amounted to $69 \%$, and the Betaproteobacteria amounted to $10 \%$ of the sequenced clones. These three groups dominated,

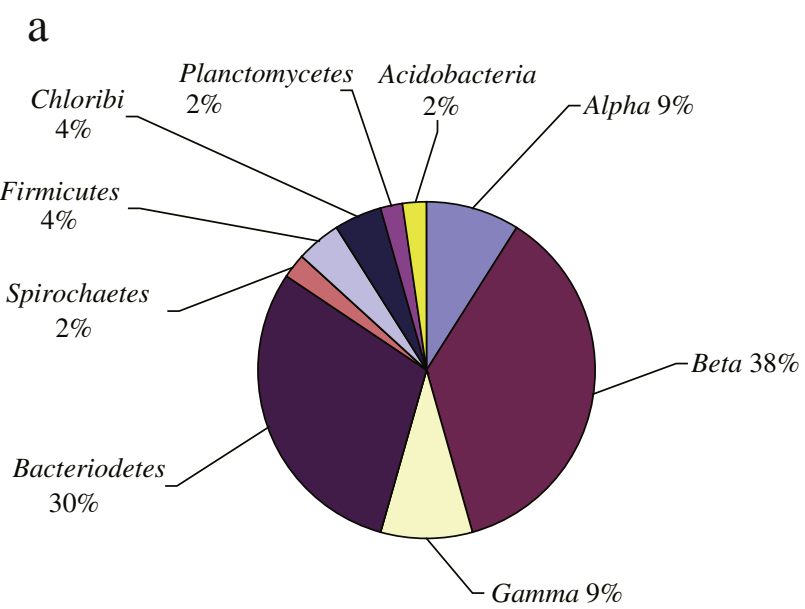

amounting to more than 79\%, in XJPW clones. This clone library also contained OTUs that were members of the Spirochaetes ( $2 \%)$, Firmicutes (7\%), Chlorobi (3\%), Chloroflexi (2\%), Epsilonproteobacteria $(2 \%)$ and Alphaproteobacteria (5\%). It was clear that a significant shift of dominant groups occurred, as ratios of Gammaproteobacteria increased from $9 \%$ to $52 \%$, Bacteriodetes decreased from $30 \%$ to $17 \%$ and Betaproteobacteria decreased from $38 \%$ to $10 \%$ for the treating community. For the minority groups, Planctomycetes (2\%) and Acidobacteria (2\%) members in the HBPW clone library were absent in the XJPW clone library while new members of Chloroflexi (2\%) and Epsilonproteobacteria (2\%) appeared in the XJ library.

Microbial diversity shift between these two libraries was also compared by phylogenetic analysis according to Proteobacteria (Fig. 2) and Nonproteobacteria (Fig. 3). 14 OTUs of 25 total from the HB clone library and 10 OTUs of 16 total from the XJPW clone library belonged to the Proteobacteria group. Among these OTUs, only 3 were found in both libraries with $\geq 97 \%$ similarity. Accordingly, 11 OTUs from the HB library and 6 OTUs from the XJPW library were classified to the Nonproteobacteria group. Only 2 OTUs were found in both libraries with $\geq 97 \%$ similarity. In total there were 5 OTUs present in both libraries with $\geq 97 \%$ similarity, accounting for 24.4\%. From both microbial composition (Figs. 2 and 3) and ratio of different groups (Fig. 1), it could be concluded that a significant shift in the sludge community had taken place in the produced water treating community. Also, the sludge community similarity $(24.4 \%)$ was in good accordance with influent community similarity (20\%) defined by DGGE.

The HBPW clone library was dominated by "Betaproteobacteria" (38\%), represented by a few OTUs of genera Thauera, Tepidicella and Tepidimonas ( $\geq 97 \%$ 16S rRNA sequence similarity). The closest representatives of these genera are thermophilic bacteria capable of utilizing halobenzoate, heavy oil or other hydrocarbon compounds associated with oil production (Song et al., 2000; Ji et al., 2011). In comparison, the XJPW clone library was dominated by Gammaproteobacteria (52\%), which only amounted to $9 \%$ in the HB library. The dominant OTUs were represented by genera Pseudidiomarina, Pseudoalteromonas and Thalassolituus ( $\geq 97 \%$ 16S rRNA sequence

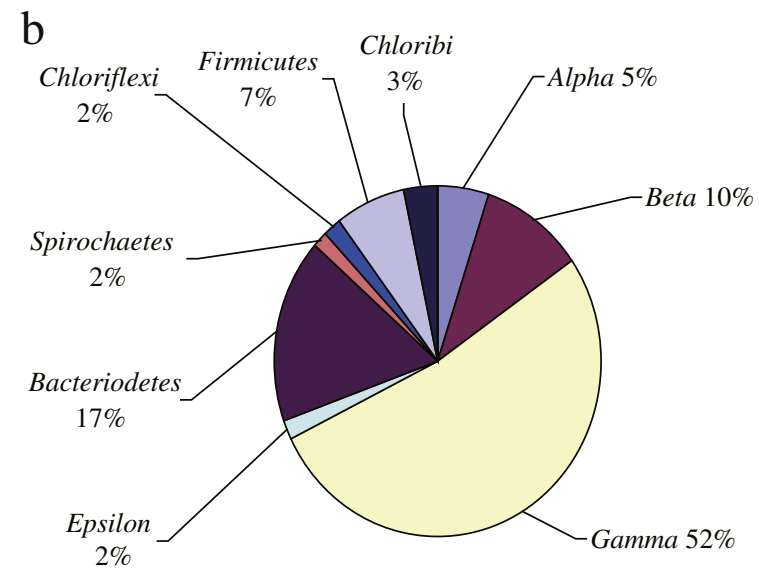

Fig. 1 - Relative abundance of groups in bacterial clone libraries of HBPW (a) and XJPW (b) sludge samples. Alpha: alpha-Proteabacteria; Gamma: gamma-Proteabacteria; Beta: bata -Proteabacteria; Epsilon: epsilon-Proteabacteria 


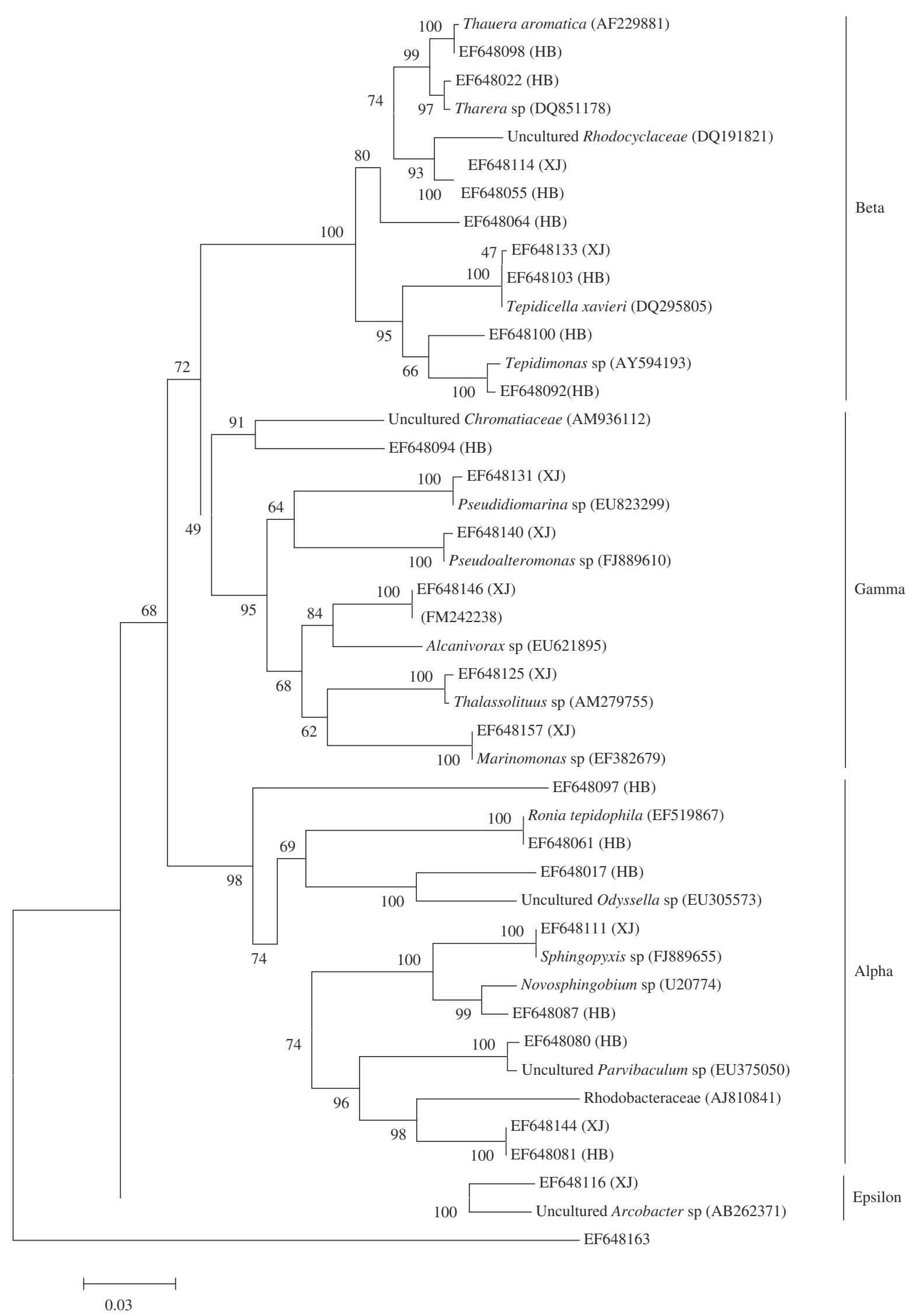

Fig. 2 - Phylogenetic tree of the Alpha, Gamma, Beta, Epsilon-Proteabacteria 16S rRNA gene phylotypes of HBPW and XJPW sequence types and closely related sequences from the GenBank database. 


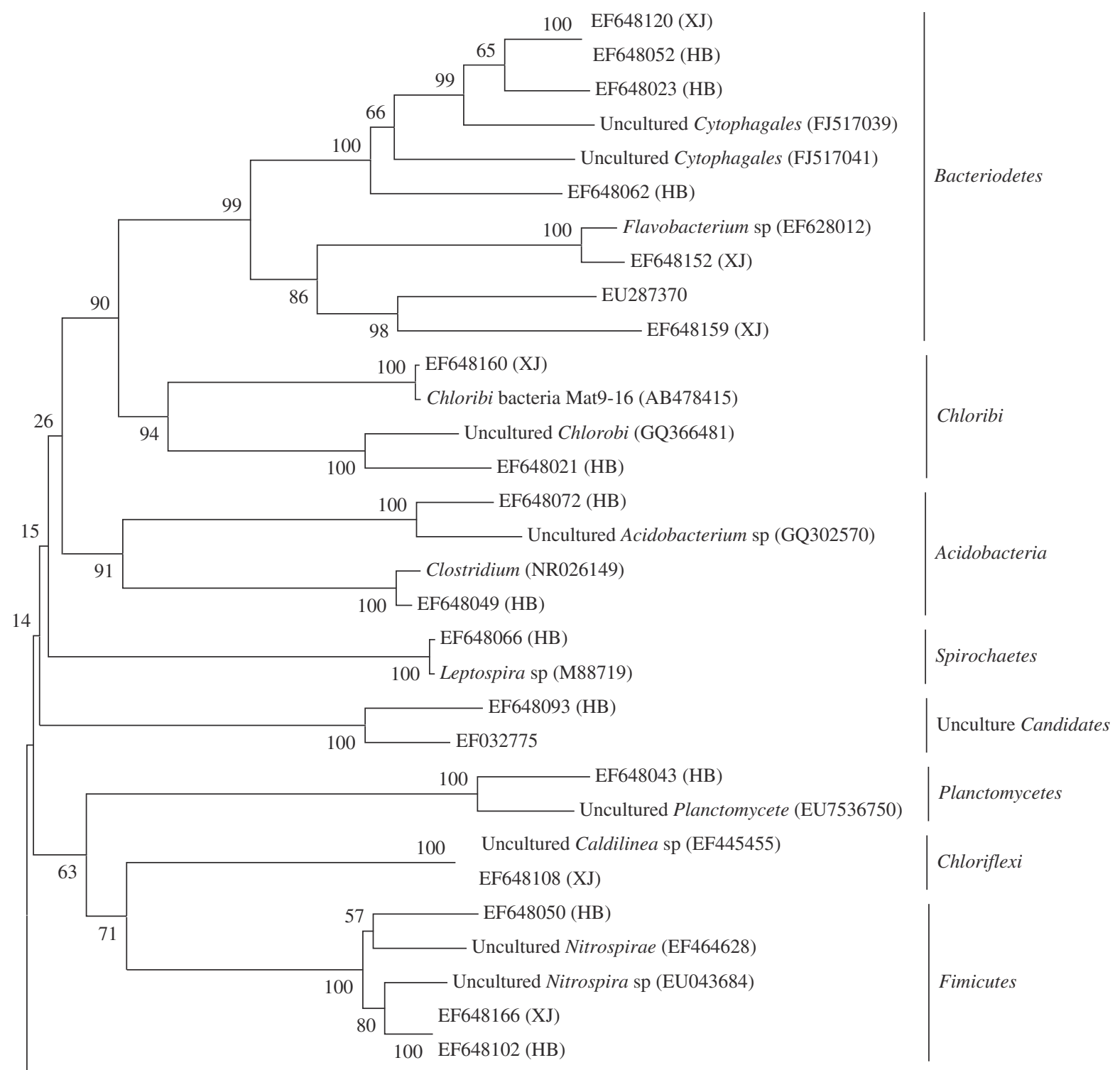

EF648163

0.03

Fig. 3 - Phylogenetic tree of the non-proteabacteria 16S rRNA gene phylotypes of HBPW and XJPW sequence types and closely related sequences from the GenBank database.

similarity). The nearest representatives of these genera are isolated from sea sediment or sea water with halotolerant or halophilic properties (Choi and Cho, 2013; Yu et al., 2010; Païssé et al., 2010). The shift of these two groups was apparently related to the different origin of the produced water generated. However, according to the database, representatives of Betaproteobacteria, the highest abundance in the HBPW clone library, are more related to catabolic properties of hydrocarbon compound degradation than those of Gammaproteobacteria, the highest abundance in the XJPW clone library, which are associated with properties of environmental living conditions. Bacteriodetes-affiliated sequences were generally distantly related to known cultivated species. Most of them were closely related to environmental clones from seawater or sediment samples. There was no obvious ratio variation for both the Alphaproteobacteria (representative of marine bacteria or clones) and Firmicutes (representative of nitrifying bacteria or clones) group between the two clone libraries. Other minor groups such as Chlorobi, Chloroflexi and Spirochaetes also remained relatively stable in these two clone libraries. The closest representatives of these sequences were associated with sulfur bacteria and marine bacteria or clones.

From these two clone libraries related to two kinds of production wastewater, some metabolic properties of the sludge community could be suggested, and more information related to community habitat or produced water origin could 

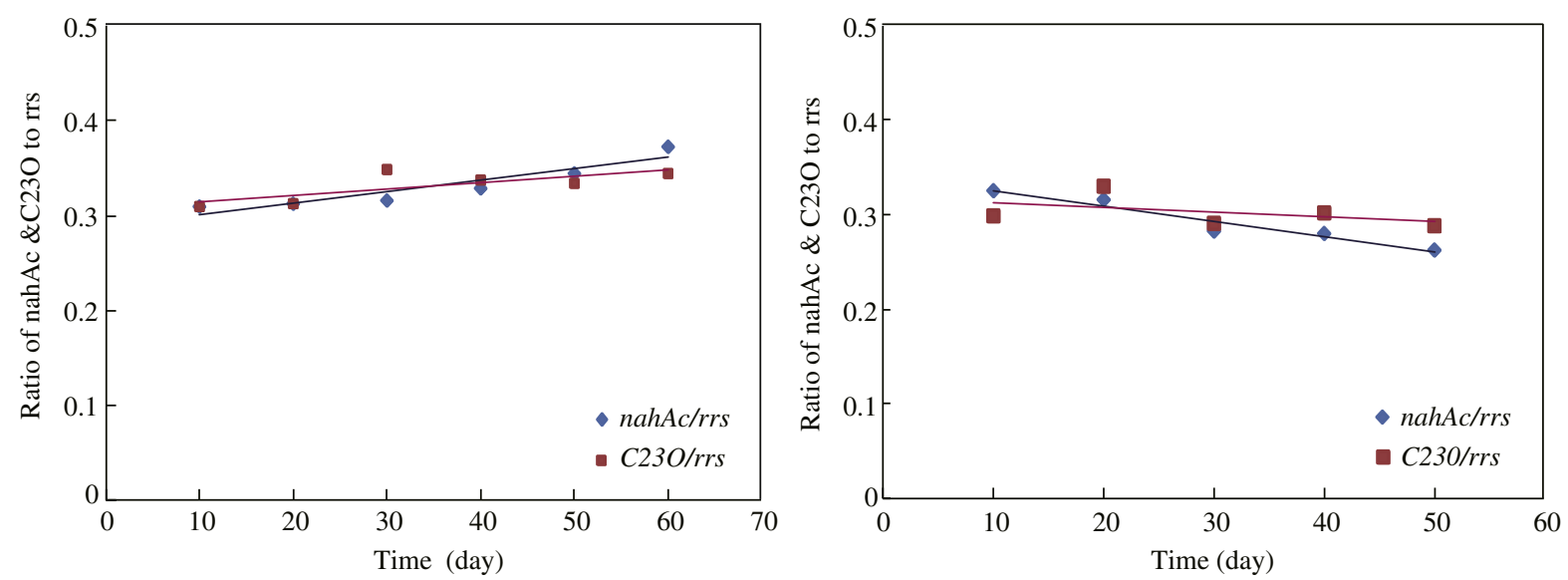

Fig. 4 - Ratio for copy number of catabolic gene to 16s rRNA gene. (a) for HBPW produced water treatment system; (b) for XJPW produced water treatment system.

be concluded. For example, more sequences from the HBPW clone library were associated with sequences from high temperature and hydrocarbon-contaminated water, which indicated that the produced water from HBPW was extracted from a high temperature oil reservoir. However, more sequences from the XJPW clone library were associated with those from high salinity environments. Interestingly, the $\mathrm{HB}$ oil field is very near the sea geographically while the XJPW oil field is located in desert, far from the sea environment. This possibly reflects the indigenous bacterial composition or geochemical characteristics in the deep oil reservoirs.

\subsection{Enumeration of the PAH catabolic genes}

Catabolic or functional genes (nahAc/phnAc and C12O/C230) encoding for key dioxygenase enzymes in catabolic pathways of LMW-PAH degradation were tracked along with rrs abundance based on PCR assay (Tuomi et al., 2004). As shown in Fig. 4, both nahAc and C230 genes in HBPW and XJPW produced water treating sludge were detected, while there were no signals of phnAc and C12O detected in the produced water treatment systems. This indicated that phnAc and C12O degradation pathways may not have occurred in the wastewater treatment systems. The ratios of $\log$ (nahAc gene copies) and $\log$ (C23O gene copies) to $\log$ (rrs copies, the level of bacterial number) were quantified to reflect the potential activity of the community for aromatic hydrocarbon removal. During each produced water treatment, the abundance of catabolic genes, nahAc and C230, was relatively stable, while it was little lower in XJPW produced water treating sludge than in HBPW produced water treating sludge. This suggested that the abundance variation of catabolic genes was associated with the potential for degradation of the target pollutant.

It is interesting that the system performed in a similar fashion in treatment of the two sources of produced water in terms of COD and LMW-PAH removal, even though a distinct shift in bacterial composition took place. After influent switching, COD removal fluctuation and a distinct sludge community shift occurred concurrently. The new compositionfunction sludge was achieved by bacterial community dispersal and assembly. The genera retrieved from both clone libraries such as Pseudoalteromonas, Marinomonas, Novosphingobium and Flavobacterium, which are regarded as the PAH degraders referred to pure cultures, were always retained in sludge (Cao et al., 2009). Most of the other species were endangered by the new environmental conditions and replaced by influent-sourced bacteria. The hypothesis is that the species loss or substitution was not equivalent to functional loss (Micheli and Halpern, 2005). It indicated that catabolic gene abundance represents structure variation more than composition or diversity variation (Debruyn and Sayler, 2009). Based on the above assumption, the conventional bioaugmentation by effective microbes or consortia should be very carefully examined.

On the other hand, for most persistent pollutants such as PAHs, the catabolic genes are often located on mobile genetic elements susceptible to high mutation rates and horizontal transfer, especially under stress (Turner et al., 2002). Also, in our previous study, it was concluded that catabolic genes encoded by plasmids contributed more than chromosomes to phenanthrene degradation (Wang et al., 2006). The transfer of catabolic plasmids between bacterial species is also possible to maintain similar function in terms of $\mathrm{PAH}$ removal. Thus the native acceptors achieve the catabolic ability and facilitate its adaptation to pollutant-contaminated sites. This is a possible reason why different bacterial communities perform similarly. This suggests an alternative strategy for soil remediation or wastewater treatment through catabolic gene mediated bioaugmentation to remove refractory organic compounds (Stuart-Keil et al., 1998).

\section{Conclusions}

In this paper, the changes in microbial community structures and abundance of catabolic genes responsible for the degradation of PAH and aromatic structures were analyzed in activated sludge systems treating two produced waters sequentially. Clone library results showed that the influent and its sourced bacteria contributed to community succession and composition in a reactor system treating hydrocarboncontaining produced waters. The existence of nahAc and C230 genes in the treating sludge communities for both produced 
waters indicated the potential removal of LMW-PAHs by the same biodegradation pathway even with an obvious shift of sludge community composition.

\section{Acknowledgments}

This study was supported by the Funds for the Creative Research Groups of China (No. 51221892) and State Hi-tech Research and Development Project of the Ministry of Science and Technology, China (No. 2012AA063401).

\section{R E F E R E N C E S}

Andrew, D.E., Lenore, S.C., Arnold, E.G., 1998. Standard Methods for the Examination of Water and Wastewater. 20th Ed. American Public Health Association, Washington, USA.

Cao, B., Nagarajan, K., Loh, K.C., 2009. Biodegradation of aromatic compounds: current status and opportunities for biomolecular approaches. Appl. Microbiol. Biotechnol. 85 (2), 207-228.

Cerniglia, C.E., 1992. Biodegradation of polycyclic aromatic hydrocarbons. Biodegradation 3, 351-368.

Chen, Y., Wang, C.X., Wang, Z.J., 2005. Residues and source identification of persistent organic pollutants in farmland soils irrigated by effluents from biological treatment plants. Environ. Int. 31 (6), 778-783.

Choi, A., Cho, J.C., 2013. Thalassolituus marinus sp. nov., a hydrocarbon-utilizing marine bacterium. Int. J. Syst. Evol. Microbiol. 63 (6), 2234-2238.

Chowdhury, K.H., Husain, T., Veitch, B., Hawboldt, K., 2009. Probabilistic risk assessment of polycyclic aromatic hydrocarbons (PAHs) in produced water. Hum. Ecol. Risk. Assess. 15 (5), 1049-1063.

Dandie, C.E., Thomas, S.M., Bentham, R.H., McClure, N.C., 2004. Physiological characterization of Mycobacterium sp. strain $1 \mathrm{~B}$ isolated from a bacterial culture able to degrade high-molecular-weight polycyclic aromatic hydrocarbons. J. Appl. Microbiol. 97 (2), 246-255.

Debruyn, J.M., Sayler, G.S., 2009. Microbial community structure and biodegradation activity of particle-associated bacteria in a coal tar contaminated creek. Environ. Sci. Technol. 43 (9), 3047-3053.

Grabowski, A., Nercessian, O., Fayolle, F., Blanchet, D., Jeanthon, C., 2005. Microbial diversity in production waters of a low-temperature biodegraded oil reservoir. FEMS Microbiol. Ecol. 4 (3), 427-443.

Hakon, D., Frøydis, G., Marit, M., Birkeland, N.K., 2008. Microbial community structure analysis of produced water from a high-temperature North Sea oil-field. A. VAN. LEEUW. 93 (1-2), 37-49.

Ji, G.D., Tan, Y.F., Zhang, L.H., 2011. Bacterial and granular sludge characteristics in an ultrahigh-temperature upflow anaerobic sludge blanket reactor treating super heavy oil-containing wastewater. Environ. Eng. Sci. 28 (2), 129-137.

Juhasz, A.L., Stanley, G.A., Britz, M.L., 2000. Degradation of high molecular weight PAHs in contaminated soil by a bacterial consortium: effects on microtox and mutagenicity bioassays. Biom. J. 4 (4), 271-284.

Kohno, T., Fukunaga, S., 1998. The 'cut-off probability' as the measure of quantal improbability in the dilution method. Water Res. 32 (10), 3099-3107.

Micheli, F., Halpern, B.S., 2005. Low functional redundancy in coastal marine assemblages. Ecol. Lett. 8, 391-400.

Païssé, S., Goñi-Urriza, M., Coulon, F., Duran, R., 2010. How a bacterial community originating from a contaminated coastal sediment responds to an oil input. Microb. Ecol. 60 (2), 394-405.

Peng, R.H., Xiong, A.S., Xue, Y., Fu, X.Y., Gao, F., Zhao, W., et al., 2008. Microbial biodegradation of polyaromatic hydrocarbons. FEMS Microbiol. Rev. 32 (6), 927-955.

Pham, V.D., Hnatow, L.L., Zhang, S.P., Fallon, R.D., Jackson, S.C., Tomb, J.F., et al., 2009. Characterizing microbial diversity in production water from an Alaskan mesothermic petroleum reservoir with two independent molecular methods. Environ. Microbiol. 11 (1), 176-187.

Song, B., Palleroni, N.J., Haggblom, M.M., 2000. Isolation and characterization of diverse halobenzoate-degrading denitrifying bacteria from soils and sediments. Appl. Environ. Microbiol. 66 (8), 3446-3453.

Stevenson, B.S., Drilling, H.S., Lawson, P.A., Dunca, K.E., Parisi1, V.A., Suflita, J.M., 2011. Microbial communities in bulk fluids and biofilms of an oil facility have similar composition but different structure. Environ. Microbiol. 13 (4), 1078-1090.

Stuart-Keil, K.G., Hohnstock, A.M., Drees, K.P., Herrick, J.B., Madsen, E.L., 1998. Plasmids responsible for horizontal transfer of naphthalene catabolism genes between bacteria at a coal tar-contaminated site are homologous to pDTG1 from Pseudomonas putida NCIB 9816-4. Appl. Environ. Microbiol. 64 (10), 3633-3640.

Tuomi, P.M., Salminen, J.M., Jorgensen, K.S., 2004. The abundance of nahAc genes correlates with the 14C-naphthanlene mineralization potential in petroleum hydrocarbon-contaminant oxic soil layers. FEMS Microbiol. Ecol. 51 (1), 99-107.

Turner, S.L., Bailey, M.J., Lilley, A.K., Thomas, C.M., 2002. Ecological and molecular maintenance strategies of mobile genetic elements. FEMS Microbiol. Ecol. 42 (2), 177-185.

Wang, Z.Y., Zhang, J., Zhang, Y., Hesham, A.E.L., Yang, M., 2006. Molecular characterization of a bacterial consortium enriched from an oilfield that degrades phenanthrene. Biotechnol. Lett. 28 (9), 617-621.

Wang, Z.Y., Li, J., Hesham, A.E.L., He, S.W., Zhang, Y., Yang, M., et al., 2007. Co-variations of bacterial composition and catabolic genes related to $\mathrm{PAH}$ degradation in a produced water treatment system consisting of successive anoxic and aerobic units. Sci. Total Environ. 373 (1), 356-362.

Yu, Y., Li, H., Zeng, Y., Chen, B., 2010. Phylogenetic diversity of culturable bacteria from Antarctic sandy intertidal sediments. Polar Biol. 33 (6), 869-875.

Zhang, Y., Sei, K., Toyama, T., Ike, M., Zhang, J., Yang, M., et al., 2008. Changes of catabolic genes and microbial community structures during biodegradation of nonylphenol thoxylates and nonylphenol in natural water microcosms. Biochem. Eng. J. 39 (2), 288-296. 